\title{
Interactive comment on "Developing the hertz art-science project to allow inaudible sounds of the Earth and Cosmos to be experienced" by Graeme J. Marlton and Juliet Robson
}

\section{Anonymous Referee \#4}

Received and published: 14 April 2020

\section{General comments}

This paper highlights a fascinating project that brings together science and art in a strong collaboration. It should prove interesting to scientists with an interest in public engagement with research, as well as artists looking to draw on science. However, I think there are some weaknesses in the paper as it is presented, which make it difficult to follow and detract from what is otherwise interesting work. It is lacking in clarity at times and I believe it would benefit from more detail at certain points.

Specific comments

There are clear connections made to interesting and relevant science content through 
the early stages of the project, during design and prototyping. The connection between research and the final installation is less clear, aside from the use of infrasound. Highlighting the scientific research content and how it was expressed in the main installation would substantially strengthen the connection to scientific questions, I think.

I would have appreciated an explication of the context of the project among related artworks. Have any other art installations used infrasound or is this the first? Are there other works that have used vibration in a similar or different way? How does this installation relate to other experiential works that incorporate scientific data? Similarly, explaining what other public engagement or artistic projects exist around infrasound or ARISE 2 would have helped site this work in the relevant landscape. Together, these would make much clearer the extent to which this work is novel.

Occasionally, the paper refers to 'playing the infrasound' (e.g. line 186, 187). I think this is a little disingenuous. The processing is quite carefully described, but my understanding of it from this is that what is actually played is synthetically generated pink noise, which is then processed according to the infrasound data. If my understanding is not quite right, then perhaps the section on the signal processing needs to be revisited. I wonder to what extent artistic licence was employed when creating the infrasound-scapes. Phrases such as 'This produced an effect that we felt was relatable to infrasound if we could hear it' suggest quite a lot, which in turn suggests a move away from the science. Perhaps the phrase 'keeping translatable authenticity' needs unpacking to clarify to what extent the experienced signals relate to the original infrasound signals. Relatedly, a flow diagram of sorts might help here (e.g. around line 152), to make clear exactly what the inputs, processes and outputs were for the prototypes and also for the final installation. Even after multiple readings of the paper, I'm still not sure I understand the relationship between the signals fed to the subwoofer and transducer - are they just the same?

The information on CTBTO stations and sensors is interesting, but I don't understand the connection between this and the project. Was data from these sensors used? Are

Printer-friendly version

Discussion paper
Interactive

comment 
the microbarometers used by CTBTO the same as the microbarometer used on this project? The connection needs to be made clear; or if there is not one, this (lines 87-91) is probably extraneous and distracting information. Likewise, the reference to playing Pink Floyd through the system is confusing - did Pink Floyd use infrasound? Or was Pink Floyd's music used somewhere in the project? If there isn't any further connection, then I would suggest it is a distracting detail.

Interactive

comment

A point that is made in passing, but that I think deserves much more attention, is that 'you had to be physically present in order to sense the frequencies, making it an immersive and experiential artwork'. The fact that the artwork could not be reproduced through audio or video recording marks it out as something special in a world that seems increasingly focussed on engaging publics with research digitally. I think perhaps more could be made of this in the wider context of public engagement with research.

On the other hand, Section 4.4 on Web and online presence comes across as rather weak. Simply stating the numbers of impressions gives no context and no conclusion. Can any analysis be done of who the Twitter followers were or who visited the website? Were they scientists? Artists? Funders? What were the most popular posts and why? How does this performance compare to similar websites or accounts? The weakness of simply stating figures is noted in the text, but if nothing further can be added to this section by way of analysis, I would consider removing it. As it stands, I think it detracts from the flow of the paper.

The assessment of feedback from the tour was also somewhat underwhelming. It seems largely to consist of sharing positive comments. This section would be much stronger if this was better contextualised. How many comments were received? How many of those were positive / negative? Can the feedback be analysed in more detail? The word cloud seems like a good start, but are there themes to be drawn out? A clearer explanation of how this feedback impacted the project would also be beneficial. On a different note, I don't think the description of the installation as "scary" needs to

Printer-friendly version

Discussion paper

3 
be considered negative, especially if part of the goal was to "re-establish links with the natural environment" including events that are "both majestic and alarming".

Much is made of the artist-scientist relationship in this work, and to my mind this (Section 5 ) is the weakest section of the paper; I would consider substantially reducing or rewriting it with a much tighter focus. A substantial portion of the text is devoted to expounding stereotypes about the differences between how scientists work and how artists work. This struck me as rather lazy writing. There are no citations of studies or research that look at this question, and I wonder what the basis is for these wideranging assertions about what scientists "will" do. Furthermore, as this paragraph progresses, it seems to lose its line of argument, and it is not clear what point is intended. Moreover, I would be wary of suggesting that the different ways two particular people react to a particular event (see lines 305-309) is as a result of one being an artist and the other a scientist - this is not a strong conclusion. Finally, the overall conclusion suggests that this collaboration "is a good model for future art science collaborations". To be more useful, I think the "model" in question needs further explanation. What was it they did that meant it worked especially well? What do other people need to know to be able to use the same model?

There are a number of grammatical and punctuation errors throughout the text that need fixing. I think it could also do with the attention of a copy editor to re-phrase a few passages as some of the writing is a little stilted. Amending these would substantially improve the readability.

Technical corrections: There were a couple of names and phrases that I think need explaining in the text. A few words of context would save me looking it up and give me a better frame of reference.

- Line 99, 'LT' is not explained - is this 'local time'?

Printer-friendly version

- Line 165, what is the Attenborough Centre - is it an art space, a science space, a community space or something else? 
- Line 211: What is 'We the Curious'?

There are some straightforward grammatical errors and misuses of punctuation

- Line 32: 'science technology engineering and maths' needs some commas

- Line 36: 'one of those was, co-author' - unnecessary comma

Interactive

- Line 41: '(Wilson 1969) see figure 1' - needs some punctuation

comment

- Line 58: 'the star in turn fluctuates in brightness, satellites like Kepler' - probably full stop, not comma

- Line 59: 'transiting exoplanet survey satellite' - this is the name of a particular satellite, treat it as such

- Line 98-99: inconsistency with spaces before 'Hz'

- Line 150: 'synthetic generated pink noise. to ensure' - capital T on 'to' Some sentences need re-phrasing, including

- Line 25: 'Technology further isolates the modern human from the natural environment in which we evolved increasingly being used as a filter through which we view the natural world.'

- Line 57: 'Sound waves move through sun stars gaseous interior because of temperature changes'

- Line 106: 'The infrasonic signals produced by the Reading thunderstorms and the infrasonic signal from the aurora is 4 times smaller.' Four times smaller than what?

- Line 107: 'This shows that different phenomena produce have different infrasound signatures'

- Line 113: 'Robson had a spare metal wheelchair made of metal that were good at transferring vibrations.' - intentional repetition of metal? 
Interactive comment on Geosci. Commun. Discuss., https://doi.org/10.5194/gc-2020-9, 2020.

GCD

Interactive comment 